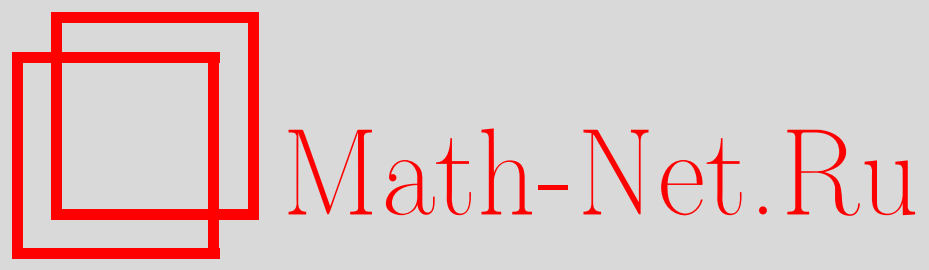

В. И. Арнольд, К лежандровой теории Штурма пространственных кривых, Функи. анализ и его прил., 1998, том 32, выпуск 2, 1-7

DOI: https://doi.org/10.4213/faa409

Использование Общероссийского математического портала Math$\mathrm{Net.Ru}$ подразумевает, что вы прочитали и согласны с пользовательским соглашением

http://www . mathnet.ru/rus/agreement

Параметры загрузки:

IP : 35.174 .16 .151

26 апреля 2023 г., 15:00:35

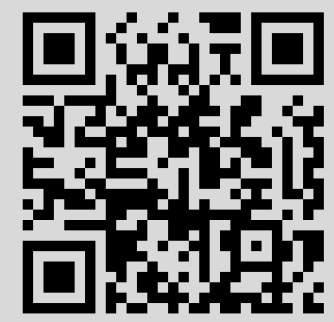


Функииональньй анализ и его приложения

1998, т. 32, вып. 2, с. 1-7

УДК 517.9

\title{
К лежандровой теории Штурма пространственных кривых ${ }^{\star}$
}

\author{
(c) 1998. В. И. Арнольд
}

\section{§1. Введение}

Теория Штурма распространяет неравенства Морса (для функций на окружности) на операторы, включающие старшие производные.

Лежандрова теория Морса (построенная Ю. В. Чекановым в 1986 г.) переносит неравенства Морса на многозначные функции (соответствующие незаузленным лежандровым подмногообразиям пространств 1-струй функций). Она является обобщением теории лагранжевых пересечений Конли, Цендера, Шаперона, Флоера, Сикорава, Лауденбаха, Хофера, Громова и др.

Ниже предпринята попытка распространить лежандрову теорию Морса на высшие производные (в том же смысле, в каком теория Штурма обобшает на высшие производные неравенство Морса).

Из теории Штурма следует существование не менее четырех точек уплощения на всякой замкнутой кривой в трехмерном проективном пространстве, имеюшей выпуклую проекцию.

Из результатов настоящей работы вытекает, в частности, что не менее четырех точек уплощения сохраняется при (не обязательно малых) деформациях стандартной кривой теории Штурма $(x=\cos t, y=\sin t, z=\cos 2 t)$, не меняющих тип лежандрова узла (образованного некоторой построенной по кривой особой лежандровой поверхностью в пятимерном многообразии контактных элементов пространства), при условии, что в процессе деформации на кривой не появляются точки нулевой кривизны.

В терминах фронта кривой (являющегося поверхностью в двойственном проективном пространстве) условия на деформацию состоят в том, что не происходит самокасаний фронта, самопересечений его ребра возврата и касаний фронта с его ребром возврата, причем ребро возврата остается неприводимым во все время деформации.

Основной идеей настоящей работы является попытка выделить в топологической теории перестроек фронтов кривых часть, не зависящую от того, что фронт связан с какой-либо кривой, справедливую для общих фронтов лежандровых многообразий (подобно тому как результаты А. В. Алексеева и В. А. Васильева о самопересечениях фронтов, полученные в начале 80-х годов и опубликованные, например, в [5], содержат многие результаты работы [6] в более общей форме [7]). 


\section{§ 2. Лежандровы оболочки кривых проективного пространства $P^{N}$ и их фронты}

Напомню основы построенной О. П. Щербаком [1] теории контактной геометрии кривой $\gamma$ в $N$-мерном проективном пространстве $P^{N}$. Ниже из этой локальной теории извлекаются глобальные топологические следствия.

Лежандровой оболочкой $L$ кривой $\gamma$ называется многообразие всех касающихся $\gamma$ контактных элементов в $P^{N}$. Это гладкое лежандрово подмногообразие многообразия $E=P T^{*} P^{N}=P T^{*}\left(P^{N}\right)^{\vee}$ контактных элементов на $P^{N}$. Точкой в $E$ является пара $(a, A)$, где $a-$ точка в $P^{N}$, а $A-$ проходящая через нее гиперплоскость. Многообразие $E$ контактных элементов на $P^{N}$ является тотальным пространством двух лежандровых расслоений (над исходным и над двойственным пространством)

$$
\rho: E \rightarrow P^{N}, \quad \rho^{\vee}: E \rightarrow\left(P^{N}\right)^{\vee} \quad\left(\text { где } \rho(a, A)=a, \rho^{\vee}(a, A)=A\right) .
$$

Фронтом $F$ кривой $\gamma$ называется проекция $F=\rho^{\vee} L$ лежандровой оболочки кривой в двойственное проективное пространство. Размерность фронта равна $N-1$.

Для кривой $\gamma$ общего положения фронт имеет лишь стандартные лежандровы особенности серии $A_{k}$ (в трехмерном пространстве - полукубические ребра возврата $A_{2}$ и ласточкины хвосты $\left.A_{3}\right)$. Он является разверткой (объединением $(N-2)$-мерных сопровождающих плоскостей) двойственной кривой $\gamma^{\vee} \subset\left(P^{N}\right)^{\vee}$ (в трехмерном пространстве - объединением ее касательных прямых). Двойственная кривая $\gamma^{\vee}$ образована соприкасающимися гиперплоскостями исходной кривой.

Единственными особенностями кривой, двойственной к кривой обшего положения в трехмерном пространстве, являются полукубические точки возврата (соответствующие точкам уплощения на исходной кривой, ласточкиным хвостам на фронте и сложенным зонтикам Уитни на развертке (объединении касательных) исходной кривой).

В окрестности такой точки исходная кривая задается параметрическими уравнениями $x=t, y=t^{2}+\ldots, z=t^{4}+\ldots$ (где отточия означают члены более высокой степени) в подходящих аффинных $\operatorname{koординатах~}(x, y, z)$. Двойственная кривая задается уравнениями $p=t^{2}+\ldots, q=t^{3}+\ldots, r=t^{4}+\ldots$ в подходящих локальных аффинных координатах двойственного пространства.

В однопараметрических семействах общего положения кривых в $P^{3}$ могут встречаться (при отдельных значениях параметра) еще два события: биуплощение кривой (степени $(1,2,5)$ вместо $(1,2,4)$ в исходном и $(3,4,5)$ вместо $(2,3,4)$ в двойственном пространстве) и перегиб (степени $(1,3,4)$ в обоих пространствах).

Соответствующие перестройки фронтов являются стандартными лежандровыми перестройками типа «бабочка» $\left(A_{4}\right)$ в первом случае и типа «клюв к клюву» $\left(A_{3}\right)$ во втором.

Мы дадим ниже оценку топологически необходимого числа точек уплощения на кривой, полученной из некоторой фиксированной кривой проективного пространства при помоши регулярной гомотопии общего положения (в терминах линий самопересечения фронта исходной кривой или в терминах линий самопересечения ее развертки и их перестроек при гомотопии). 


\section{§3. Штурмовость кривой в проективном пространстве}

Ориентируем проективное пространство $P^{N}, N=2 n+1$. В окрестности точки уплощения параметризованная кривя общего положения задается уравнениями вида

$$
x_{1}=t, \quad x_{2}=t^{2}+\ldots, \quad \ldots, \quad x_{N-1}=t^{N-1}+\ldots, \quad x_{N}=t^{N+1}+\ldots
$$

в некоторой системе аффинных координат с началом в этой точке.

ОПРедЕлЕниЕ. Точка уплощения называется положительной, если эта система координат ориентирует $P^{N}$ правильно (и отричательной в противном случае).

Знаки последовательных точек уплощения на кривой общего положения чередуются.

ЗАМЕЧАНИЕ. Знаки можно было бы определять и при помощи двойственной кривой. При изменении направления обхода кривой знаки всех ее точек уплощения умножаются на $(-1)^{n}$.

Число точек уплошения замкнутой кривой в нечетномерном проективном пространстве четно, так как при переходе через точку уплощения меняется знак сопровождающего репера.

На множестве точек уплощения кривой общего положения в нечетномерном проективном пространстве действует инволюция без неподвижных точек: у каждой точки уплощения есть связанная с ней точка.

Связь объясняется тем, что в каждой вершине ласточкиного хвоста заканчивается линия самопересечения фронта, начинаюшаяся в вершине другого ласточкиного хвоста.

ОПРЕДЕЛЕНИЕ. Связанной парой вершин ласточкиных хвостов на фронте общего положения, лежащем в трехмерном многообразии, называется пара концевых точек линии самопересечения фронта (на этой линии могут быть точки возврата и точки пересечения двух не касающихся друг друга ветвей линии самопересечения).

ЗАмЕчАнИЕ. Аналогичным образом определяются связанные пары вершин ласточкиных хвостов на фронтах в $(2 n+1)$-мерных многообразиях, имеющих лишь особенности типа $A_{k}$.

В окрестности вершины «ласточкиного хвоста $A_{2 n+1}$ » фронт диффеоморфен многообразию многочленов $x^{2 n+2}+\lambda_{1} x^{2 n}+\cdots+\lambda_{2 n+1}$, имеющих кратный вещественный корень. В вершине $(\lambda=0)$ заканчивается кривая многочленов $2 A_{n}$ вида $\left(x^{2}-a^{2}\right)^{n+1}$.

ОПРЕДЕЛЕНИЕ. Две точки уплощения кривой общего положения в нечетномерном проективном пространстве называются связанной парой, если соответствующие им вершины ласточкиных хвостов на фронте образуют связанную пару.

ЗАмЕчАниЕ. В исходном трехмерном пространстве связанная пара точек уплощения соединена отрезком линии самопересечения развертки исходной кривой. Это видно из того, что пересечение в точке $q \in P^{3}$ касательных $l_{a}$ и $l_{b}$ кривой $\gamma$ в точках $a$ и $b$ определяет плоскость $Q$, проходящую через $l_{a}$ и $l_{b}$. Эта плоскость является точкой $Q \in\left(P^{3}\right)^{\vee}$ в двойственном пространстве, содержащейся в прямых $L_{A}$ и $L_{B}$ двойственного пространства, каса- 
ющихся двойственной кривой $\gamma^{\vee}$ в соответствующих $a$ и $b$ точках $A$ и $B$. А именно, прямая $L_{A}$ состоит из всех плоскостей исходного пространства, касательных к $\gamma$ в $a$, а $L_{B}-$ в $b$.

Таким образом, точка $q$ самопересечения развертки кривой $\gamma$ (т.е. точка самопересечения фронта двойственной к $\gamma$ кривой $\gamma^{\vee}$ ) соответствует определенной точке $Q$ самопересечения фронта $F$ кривой $\gamma$ (т. е. точке самопересечения развертки двойственной к $\gamma$ кривой $\left.\gamma^{\vee}\right)$.

Когда точка $q$ пробегает отрезок линии самопересечения развертки исходной кривой, соединяющий две точки уплощения, точка $Q$ пробегает отрезок линии самопересечения фронта между вершинами соответствующих ласточкиных хвостов.

ОСНОВНОЕ ОПРЕДЕЛЕНИЕ. ШІтурмовостью $s$ кривой общего положения в нечетномерном проективном пространстве называется число связанных пар точек уплошения одного знака.

ЗАмЕчАниЕ. Штурмовость любой замкнутой кривой четна, так как число положительных пар равно числу отрицательных.

ПримеР. Штурмовость стандартной кривой теории Штурма в $P^{2 n+1}$

$$
\left(x_{1}=\cos t, y_{1}=\sin t, \ldots, x_{n}=\cos n t, y_{n}=\sin n t, z=\cos (n+1) t\right),
$$

где $n$ нечетно, есть $s=n+1$ (связанные точки противоположны).

\section{§4. Оценка числа точек уплощения снизу}

Рассмотрим гладкую замкнутую кривую $\gamma$ общего положения, вложенную в трехмерное проективное пространство (мы предполагаем, что кручение кривой везде отлично от 0 , исключая конечное число простейших точек уплощения, где кручение имеет нуль первого порядка).

ОПРЕДЕлЕНИЕ. Регулярная гомотопия кривой $\gamma$ называется допустимой, если она является регулярной гомотопией общего положения с трансверсальным прохождением отдельных моментов биуплощения, но без моментов перегиба и, кроме того, фронт деформируемой кривой не испытывает а) самокасаний, б) самопересечений ребра возврата, в) касаний ребра возврата фронта с фронтом.

ЗАмечАниЕ. Самокасание фронта означает самопересечение исходной кривой. Самопересечение ребра возврата фронта означает самопересечение двойственной кривой (и самокасание развертки исходной кривой). Касание ребра возврата фронта с фронтом означает касание исходной кривой с одной из своих соприкасающихся плоскостей (причем точка касания лежит на касательной к кривой в точке соприкосновения), т. е. ее касание со своей разверткой. В момент прохождения перегиба ребро возврата фронта было бы приводимо (содержало бы прямую).

Теорема. При допустимых гомотопиях кривой ее штурмовость не меняется.

СлЕДСТВИЕ. Число точек уплощения кривой в прочессе допустимых гомотопий никогда не может стать меньше удвоенной штурмовости исходной кривой. 
ЗАмЕЧАНИЕ. Число $2 s$, оценивающее снизу число точек уплощения, делится на 4.

ДокАЗАТЕльство. Список перестроек в типичных однопараметрических семействах фронтов лежандровых отображений (в пространствах не слишком большой размерности) был найден в [2]. О. П. Щербак доказал в [1], что перестройки фронтов типичных однопараметрических семейств кривых в трехмерном проективном пространстве составляют следующую часть этого списка.

I. Перестройки с изменением числа ласточкиных хвостов:

1. $A_{4}$ («бабочка» по терминологии Тома): рождение или смерть связанной пары близких вершин ласточкиных хвостов на ребре возврата фронта (эти вершины соединены малым отрезком линии самопересечения фронта, имеющим две точки возврата).

2. $A_{3}$ («клюв к клюву» по терминологии Тома): рождение или смерть пары близких вершин ласточкиных хвостов, далеких друг от друга вдоль ребра возврата.

II. Перестройки, не меняюшие число ласточкиных хвостов.

3. Самокасание фронта (морсовская перестройка следа, оставленного одной гладкой ветвью фронта на другой).

4. Касание ребра возврата фронта с гладкой частью фронта (рождение или смерть двух точек возврата линии самопересечения фронта).

5. Самопересечение ребра возврата фронта (переход двух точек возврата линии самопересечения фронта с одной ветви ребра возврата фронта на другую).

6. Прохождение гладкой ветви фронта через вершину ласточкиного хвоста (с рождением или смертью пары точек возврата на линии самопересечения фронта).

7. Прохождение ребра возврата фронта через линию пересечения двух его гладких ветвей (с прохождением этой линии пересечения через точку возврата другой ветви линии самопересечения фронта).

8. Прохождение четырех гладких ветвей фронта через одну точку (при котором каждая из шести линий пересечения двух ветвей меняется гладко).

9. Касание линии самопересечения фронта с гладкой частью фронта (при котором каждая из трех линий пересечения двух ветвей фронта меняется гладко).

Докажем, что при перестройках, происходящих в процессе допустимой гомотопии, штурмовость не меняется. Действительно, перестройки 6, 7, 8,9 не меняют ни связанных пар, ни знаков ласточкиных хвостов.

Перестройки 3,4 и 5 запрещены условием допустимости гомотопии.

Перестройка 1 происходит в момент биуплощения. В этот момент рождается или умирает связанная пара (соседних на кривой) точек разных знаков, которые соединены на фронте (или на развертке) линией самопересечения. Поэтому штурмовость при этой перестройке не меняется.

Перестройка 2 происходит только в момент перегиба. Поскольку мы исключили прохождение перегибов из числа допустимых гомотопий, теорема доказана.

Доказательство показывает также, как меняется штурмовость при недопустимых перестройках $(2,3,4,5)$. Поэтому можно оценить штурмовость 
(и, значит, число точек уплощения на кривой) через штурмовость на исходной кривой и число недопустимых перестроек.

ЗАмЕчАНИЕ. При прохождении точки перегиба на кривой рождаются или умирают две соседние точки уплощения разных знаков, но они, вообще говоря, не образуют связанной пары. Если связанные с умирающими точками уплощения точки имеют (соответственно) такие же знаки, как сами эти точки, то штурмовость кривой уменьшается на два при их умирании.

Если же хотя бы одна из связанных с ними точек имеет знак, отличный от знака своей пары, то штурмовость при такой перестройке сохраняется. Прохождение таких точек перегиба можно было бы разрешить при определении допустимых гомотопий.

Точно так же можно было бы допустить «эллиптические» перестройки 3 , 4 и 5 (при прохождении которых рождается или умирает маленькая линия самопересечения фронта) - они не меняют штурмовости.

Можно также усилить результаты, различая стягиваемые и нестягиваемые к кривой отрезки линии самопересечения развертки между связанными точками уплощения.

\section{§5. Примеры}

ПРИмеР 1. Рассмотрим пространственную кривую, заданную в цилиндрических координатах уравнениями $r=1-a \cos \varphi, z=\sin 2 \varphi$. При увеличении параметра $a$ от 0 до $1 / 2$ на этой кривой имеются четыре точки уплощения, две из которых сливаются (в точке перегиба $\varphi=0)$ при $a=1 / 2$.

При $a<1 / 2$ кривая имеет квадратично выпуклую проекцию и две перемежающиеся связанные пары точек уплощения знаков $(++)$ и $(--)$, так что штурмовость равна 2 .

При переходе $a$ через $1 / 2$ происходит перестройка 2. Штурмовость уменьшается на 2, число точек уплощения становится равным 2. В частности, полученная кривая не имеет выпуклых проекций (ни из какой точки проективного пространства), так как кривая с выпуклой проекцией имеет не меньше четырех точек уплошения [3, 4].

Этот пример показывает, что разрешить прохождение точек перегиба ни в теореме, ни в следствии нельзя, по крайней мере если связанные с исчезающими уплощения имеют тот же знак.

ПримеР 2. Рассмотрим пространственную кривую, заданную уравнениями $x=\cos \varphi, y=\sin \varphi, z=\cos 3 \varphi$. Эта кривая имеет шесть точек уплощения. Линия самопересечения развертки состоит из трех отрезков прямых, соединяющих противоположные точки уплощения «через бесконечность», и бесконечно удаленной прямой $z=0$. В точках пересечения радиальных прямых с бесконечно удаленной происходит морсовское касание обеих гладких ветвей развертки.

При малом шевелении $(z=\cos 3 \varphi+\varepsilon f(\varphi))$ можно перестроить линию самопересечения в гладкую кривую, так что последовательные точки уплощения будут образовывать связанные пары $(+-)(+-)(+-)$ (например, можно взять $f=\sin 6 t)$. Штурмовость полученной кривой равна нулю. Наша теорема не гарантирует наличия точек уплощения, хотя кривая и имеет выпуклую проекцию. 
Развертка кривой с выпуклой проекцией может рассматриваться как двулистное сечение одномерного расслоения над листом Мёбиуса (дополнительным к выпуклой области на проективной плоскости, ограниченной проекцией кривой).

Проекции линий самопересечения развертки делят лист Мёбиуса на части. Эти части можно разделить на «положительные» и «отрицательные», так что знак меняется при каждом пересечении проекций линий самопересечения развертки.

Если эти части ориентируемы, то штурмовость кривой не меньше двух, а число точек уплощения не меньше четырех (и остается не меньшим четырех при допустимых гомотопиях).

Пример 2 показывает, что возможна неориентируемая часть (и нулевая штурмовость).

ПримеР 3. Погруженная кривая $(x=\sin 2 t, y=\cos 2 t, z=\cos 4 t)$ имеет 8 точек уплощения. При малом шевелении, делающем ее вложенной, она превращается в кривую общего положения со штурмовостью 4. Эта кривая не имеет выпуклых проекций (так как проходящие через ось z плоскости, пересекающие ее более чем в двух точках, заметают все проективное пространство).

ЗАмечАниЕ. Вероятно, теорему можно распространить на более общий случай фронтов лежандровых многообразий, не связанных с кривыми, лишь бы ласточкины хвосты разделялись на положительные и отрицательные. С кривой связано также лежандрово многообразие с особенностями, являющееся объединением лежандровых оболочек данной и двойственной кривых. Нормальные формы этих особенностей и их перестроек, кажется, до сих пор не описаны в контактной геометрии, как это должно бы было быть сделано.

\section{ЛитературА}

1. Щербак О. П. Проективно двойственные пространственные кривые и лагранжевы особенности. Труды Тбилисского унив., 232/233, 280-336 (1982).

2. Arnold V. I. Wave front evolution and equivariant Morse lemma. Comm. Pure Appl. Math., 29, No. 6, 557-582 (1976).

3. Arnold V. I. On the number of flattening points on space curves. in: Ya. G. Sinai's Moscow Seminar on Dynamical System, Am. Math. Soc. Transl., Ser. 2, Vol. 171, Providence, 1995, pp. 11-22.

4. Barner M. Über die Mindestanzahl stationären Schmeigebenen bei geschlossenen strengkonvexen Raumkurven, Abh. Math. Sem. Univ. Hamburg, 20, 196-215 (1956).

5. Arnold V. Singularities of Caustics and Wave Fronts, Kluwer, Dordrecht, 1990 ( 55.2 , p. 100).

6. Nun̆o Ballesteros J. J., Romero Fuster M. C. Global bitangency properties of generic closed space curves, Math. Proc. Cambridge Philos. Soc., 112, 519-526 (1992).

7. Васильев B. A. Самопересечения волновых фронтов и лагранжевы (лежандровы) характеристические числа. Функц. анализ и его прил., 16, вып. 2, 68-69 (1982).

Математический институт им. В. А. Стеклова РАН Universite Paris-Dauphine, CEREMADE 